\title{
Racial and socioeconomic disparities in the selection of prostate brachytherapy
}

\author{
David Schreiber, MD², Shan-Chin Chen, MD², Justin Rineer, MD³, Jeffrey Weiss, MD'.4, Marvin Rotman, MD², \\ David Schwartz, MD'.2 \\ IDepartment of Veterans Affairs, New York Harbor Healthcare System, Brooklyn, New York, 2Department of Radiation Oncology. \\ SUNY Downstate Medical Center, Brooklyn, New York, ${ }^{3}$ MD Anderson Cancer Center Orlando, Orlando, Florida, ${ }^{4}$ Department of Urology \\ SUNY Downstate Medical Center, Brooklyn, New York, USA
}

\begin{abstract}
Purpose: To utilize the surveillance, epidemiology, and end results database to analyze whether there are racial or socioeconomic disparities associated with the selection of prostate brachytherapy.

Material and methods: We selected patients who were diagnosed with localized prostate cancer between 2004-2006 and who underwent treatment with radiation. Data regarding race and estimates of socioeconomic status were also obtained by analyzing the average reported cost of living adjusted income in the SEER county from which the patient was treated, and dividing these results into quartiles. Multivariate logistic regression analysis was used to determine whether there were any disparities associated with brachytherapy use.

Results: A total of 38704 patients were included in the analysis. Most patients (57\%) received EBRT alone, while the remaining $43 \%$ of patients had brachytherapy as a component of their treatment, either alone (30.2\%) or in combination with EBRT (12.2\%). On multivariate logistic regression, prostate brachytherapy use was less likely in African American patients with an odds ratio of 0.89 (95\% CI: $0.84-0.95, p<0.001)$, and was more likely to be used in those with higher socioeconomic status. Regarding socioeconomic status, the odds ratio for receiving brachytherapy was 1.65 (95\% CI: 1.551.75) for the $25-50 \%$ quartile, 1.92 (95\% CI: 1.81-2.04) for the 50-75\% quartile, and 2.05 (95\% CI: $1.93-2.18)$ for the $75-100 \%$ quartile, respectively $(p<0.001)$.

Conclusions: There do appear to be socioeconomic and racial disparities in the selection of prostate brachytherapy. These findings may have both significant equality of care as well as cost of care implications.

Key words: brachytherapy, prostate cancer, racial disparities, radiation, socioeconomic disparities.

\section{Purpose}

Socioeconomic status has been observed to be a prognostic factor in a wide variety of adult cancers in the United States, among which is prostate cancer, the most prevalent malignancy among American men. It is estimated that in 2013, there will be 238590 new diagnoses of prostate cancer with 29720 dying from this disease [1]. African Americans and those of lower socio-economic status have historically been reported to have a higher incidence of disease and greater mortality [2,3]. A previously published surveillance, epidemiology, and end results (SEER) database analysis of 98484 Californian men highlighted the effect of socioeconomic disparities and found an association between higher socioeconomic states with higher incidence of prostate cancer diagnosis, and lower rates of mortality. However, even adjusting for socioeconomic differences, the influence of race could not be discounted, as African American men had a significantly higher burden of death due to prostate cancer [4]. Additionally, socioeconomic difference can imply a multitude of other disparities that impact clinical outcome. Poverty can correlate with a lack of adequate insurance coverage, poor follow-up, delay of diagnosis, or more advanced stage of presentation [5].

Even when treatment is given, there may be significant differences in the treatment modalities that are either selected by or offered to those of different racial and socioeconomic status [6]. A 1999 multi-institutional survey of 36496 patient records revealed a significant increase in brachytherapy use over a 10 year period from 1989-1999 [7], and a more recent study based in Europe mimicked these findings [8]. However, these overall trends in treatment are not necessarily homogeneous across all patient demographics. Analysis of SEER data have revealed increasing use of radiation therapy as a definitive treatment modality in patients 65 years of age or older, but not younger patients [9]. In this report, we analyze the SEER database to search for such disparities in treatment selection, specifically for prostate brachytherapy. 


\section{Material and methods}

We identified patients with clinically localized prostate adenocarcinoma and negative lymph nodes who were diagnosed between 2004-2006. Patients were selected for further analysis if they were treated with definitive external beam radiation (EBRT), external bream radiation plus brachytherapy (EBRT + brachy) or brachytherapy alone. We excluded patients who were treated with radical prostatectomy or of they received any other treatment aside from definitive radiotherapy for the treatment of their prostate cancer. In all patients, complete data were available regarding their tumor, node, metastasis (TNM) staging, as well as their pre-treatment prostate-specific antigen (PSA), and Gleason primary and secondary scores. This information was used to broadly group patients into National Comprehensive Cancer Network (NCCN) risk groupings. Per the NCCN risk groupings, low risk disease represents patients with clinical stage $\mathrm{T} 1-\mathrm{T} 2 \mathrm{a}, \mathrm{PSA} \leq 10 \mathrm{ng} / \mathrm{mL}$, and Gleason 6 disease. Intermediate risk represents patients with clinical stage T2b-c, PSA 10.1-20 ng/mL or Gleason 7 disease. High risk represents patients with clinical stage T3, PSA > $20 \mathrm{ng} / \mathrm{mL}$ or Gleason 8-10 disease. Data regarding race and socioeconomic status were also collected. Socioeconomic status was estimated using the SEER database by analyzing the average reported cost of living adjusted income in the SEER county from which the patient was treated, and dividing these results into quartiles.

Descriptive and statistical analyses were performed to analyze whether there were any racial or socioeconomic disparities in regards to the incorporation of brachytherapy into the treatment paradigm. Pearson chi square analysis was used to compare all groups. Pair-wise analysis was used to compare groups with more than two variables. In order to control for possible confounding between race and socioeconomic status, multivariate logistic regression analysis was performed to evaluate the potential impact of race, socioeconomic status, and NCCN risk group on the selection of prostate brachytherapy. Statistical significance was defined as a $p$-value $<0.05$. SPSS version 20.0 was used to perform all analyses.

\section{Results}

A total of 38704 patients met the selection criteria. Of these, 15213 (39.3\%) had low risk disease, 15678 (40.5\%) had intermediate risk disease, and 7813 (20.2\%) had high risk disease. Most patients (57\%) received EBRT alone, while the remaining $43 \%$ of patients had brachytherapy as a component of their treatment, either alone $30.2 \%$ ) or in combination with EBRT (12.2\%). For patients with low risk disease, brachytherapy was used in $56 \%$ of the time. This decreased to $39 \%$ for those with intermediate risk disease, and $25.5 \%$ for those with high risk disease. When analyzing the entire cohort, there was an overall trend for increased brachytherapy use in patients of Caucasian race, lower risk disease and higher socioeconomic status (Table 1). When controlling for NCCN risk group alone, there remained a highly significant disparity in brachytherapy use with increasing socioeconomic quartiles except for the comparisons between the two higher quartiles for low and intermediate risk disease (Table 2). Otherwise, brachytherapy use appeared to consistently increase with improving socioeconomic status. There was a smaller racial disparity as well, with Caucasians appearing to be more likely to undergo brachytherapy, though this difference appeared to decrease with increasing risk group.

On multivariate logistic regression analysis, these trends remained consistently highly significant. Prostate brachytherapy was more likely to be used in Caucasians, people of lower risk disease, and those with higher socioeconomic status ( $p<0.001$ for all comparisons). As can be seen in Table 3, brachytherapy was used less often with higher risk groups. African Americans appeared to be less likely to undergo brachytherapy with a HR of 0.89 (95\% CI: 0.84$0.95, p<0.001)$. In addition, there was a consistent and highly significant trend of increased brachytherapy use that corresponded to improving socioeconomic status, with a HR of 1.65 (95\% CI: 1.55-1.75), 1.92 (95\% CI: 1.81-2.04), 2.05 (95\% CI:

Table 1. Comparison of brachytherapy use as a component of treatment for the entire cohort

\begin{tabular}{|c|c|c|c|c|}
\hline & EBRT & Brachy & $p$-value & Pair-wise comparison \\
\hline Race & & & $<0.001$ & $\mathrm{~N} / \mathrm{A}$ \\
\hline Caucasian $(n=33$ 425) & $56.4 \%$ & $43.6 \%$ & & \\
\hline African American $(n=5279)$ & $60.9 \%$ & $39.1 \%$ & & \\
\hline NCCN groups & & & $<0.001$ & $<0.001$ for all comparisons \\
\hline $\operatorname{Low}(n=15213)$ & $43.9 \%$ & $56.1 \%$ & & \\
\hline Intermediate $(n=15678)$ & $61 \%$ & $39 \%$ & & \\
\hline $\operatorname{High}(n=7813)$ & $74.5 \%$ & $25.5 \%$ & & \\
\hline Economic Status & & & $<0.001$ & $<0.001$ unless otherwise noted \\
\hline $1^{\text {st }}(0-25 \%)$ quartile $(n=8815)$ & $68.5 \%$ & $31.5 \%$ & & \\
\hline $2^{\text {nd }}(25-50 \%)$ quartile $(n=9109)$ & $57.1 \%$ & $42.9 \%$ & & \\
\hline $3^{\text {rd }}(50-75 \%)$ quartile $(n=10076)$ & $52.8 \%$ & $47.2 \%$ & & 0.008 comparing quartile 3 and 4 \\
\hline $4^{\text {th }}(75-100 \%)$ quartile $(n=10703)$ & $51.3 \%$ & $48.7 \%$ & & \\
\hline
\end{tabular}

EBRT - external beam radiation therapy, Brachy - brachytherapy, NCCN - National Cancer Care Network 
Table 2. Comparison of brachytherapy use as a component of treatment stratified by NCCN risk group

\begin{tabular}{|c|c|c|c|c|}
\hline & EBRT & Brachy & $p$-value & Pair-wise comparison ( $p$ value) \\
\hline \multicolumn{5}{|c|}{ NCCN Low Risk } \\
\hline Race & & & $<0.001$ & N/A \\
\hline Caucasian $(n=13,266)$ & $43 \%$ & $57 \%$ & & \\
\hline African American $(n=1,947)$ & $49.6 \%$ & $50.4 \%$ & & \\
\hline Economic status & & & $<0.001$ & $<0.001$ all comparisons unless otherwise noted \\
\hline $1^{\text {st }}(0-25 \%)$ quartile $(n=3,230)$ & $57.4 \%$ & $42.6 \%$ & & \\
\hline $2^{\text {nd }}(25-50 \%)$ quartile $(n=3,505)$ & $43.1 \%$ & $56.9 \%$ & & \\
\hline $3^{\text {rd }}(50-75 \%)$ quartile $(n=4,143)$ & $39.5 \%$ & $60.5 \%$ & & 0.373 comparing quartile $3 \& 4$ \\
\hline $4^{\text {th }}(75-100 \%)$ quartile $(n=4,334)$ & $38.5 \%$ & $61.5 \%$ & & \\
\hline \multicolumn{5}{|c|}{ NCCN Intermediate Risk } \\
\hline Race & & & 0.01 & N/A \\
\hline Caucasian $(n=13,493)$ & $60.6 \%$ & $39.4 \%$ & & \\
\hline African American $(n=2,185)$ & $63.4 \%$ & $36.6 \%$ & & \\
\hline Economic status & & & $<0.001$ & $<0.001$ all comparisons unless otherwise noted \\
\hline $1^{\text {st }}(0-25 \%)$ quartile $(n=3,669)$ & $71.8 \%$ & $28.2 \%$ & & \\
\hline $2^{\text {nd }}(25-50 \%)$ quartile $(n=3,754)$ & $61.3 \%$ & $38.7 \%$ & & \\
\hline $3^{\text {rd }}(50-75 \%)$ quartile $(n=4,025)$ & $56.5 \%$ & $43.5 \%$ & & 0.412 comparing quartile $3 \& 4$ \\
\hline $4^{\text {th }}(75-100 \%)$ quartile $(n=4,230)$ & $55.6 \%$ & $44.4 \%$ & & \\
\hline \multicolumn{5}{|c|}{ NCCN High Risk } \\
\hline Race & & & 0.42 & N/A \\
\hline Caucasian $(n=6,666)$ & $74.3 \%$ & $25.7 \%$ & & \\
\hline African American $(n=1,147)$ & $75.4 \%$ & $24.6 \%$ & & \\
\hline Economic status & & & $<0.001$ & $<0.001$ all comparisons \\
\hline $1^{\text {st }}(0-25 \%)$ quartile $(n=1,916)$ & $80.8 \%$ & $19.2 \%$ & & \\
\hline $2^{\text {nd }}(25-50 \%)$ quartile $(n=1,850)$ & $75.2 \%$ & $24.8 \%$ & & \\
\hline $3^{\text {rd }}(50-75 \%)$ quartile $(n=1,908)$ & $73.8 \%$ & $26.2 \%$ & & \\
\hline
\end{tabular}

NCCN - National Cancer Care Network

1.93-2.18), for each increase in socioeconomic quartile, respectively $(p<0.001)$.

\section{Discussion}

In this large population-based study of more than 38000 patients who elected treatment with radiation, there are highly significant differences in the selection of brachytherapy associated with socioeconomic, and to somewhat lesser degree racial factors. We found there to be relatively small, but significant racial differences in the selection of brachytherapy, particularly for low and intermediate risk disease, with African-American patients being less likely to undergo brachytherapy than Caucasians. There have been several conflicting reports regarding the possibility of racial disparities in the selection of definitive treatment for prostate cancer. For example, a prior National Cancer Institute analysis of several cancer registries from 1998 to 2002 looked at the rates of radical prostatectomy, external beam radiation, and/or brachytherapy among different racial groups. That cohort study suggested that younger, intermediate/high-risk African-American men appear less
Table 3. Multivariate logistic regression analysis of the use of prostate brachytherapy while accounting for race, NCCN risk group, and socioeconomic status

\begin{tabular}{|c|c|c|c|}
\hline & Odds ratio & $95 \% \mathrm{Cl}$ & $p$-value \\
\hline \multicolumn{4}{|l|}{ Race } \\
\hline Caucasian & 1 & & \\
\hline African American & 0.89 & $0.84-0.95$ & $<0.001$ \\
\hline \multicolumn{4}{|l|}{ NCCN Risk Group } \\
\hline Low & 1 & & \\
\hline Intermediate & 0.50 & $0.48-0.53$ & $<0.001$ \\
\hline High & 0.27 & $0.25-0.29$ & $<0.001$ \\
\hline \multicolumn{4}{|l|}{ Socioeconomic status } \\
\hline $1^{\text {st }}(0-25 \%)$ quartile & 1 & & \\
\hline $2^{\text {nd }}(25-50 \%)$ quartile & 1.65 & $1.55-1.75$ & $<0.001$ \\
\hline $3^{\text {rd }}(50-75 \%)$ quartile & 1.92 & $1.81-2.04$ & $<0.001$ \\
\hline $4^{\text {th }}(75-100 \%)$ quartile & 2.05 & $1.93-2.18$ & $<0.001$ \\
\hline
\end{tabular}

NCCN - National Cancer Care Network, 95\% Cl-95\% confidence interval 
likely to receive definitive therapy compared to non-Hispanic Caucasian men [10]. A SEER analysis by Underwood et al. reached similar conclusions [11]. In contrast, in a large study from the Massachusetts cancer registry encompassing $>21,000$ patients diagnosed with prostate cancer between 1998-2002, while there were racial disparities in the selection of surgery, there were no racial differences in the selection of prostate brachytherapy even on multivariable analysis [12]. Similarly, a smaller report from the North Carolina Health Care Access Project encompassing 811 men diagnosed between 2004-2007, also revealed no significant racial differences in the selection of prostate brachytherapy. However, both of these latter comparisons have significantly fewer patient numbers than the 38000 patients included in this study. In the study from the Massachusetts cancer registry, while 21000 patients were analyzed, roughly 8000 of them received radiation. Similarly, in the study from North Carolina, roughly 300 patients received radiation.

Similarly, we found in this study that there are socioeconomic differences in the selection of brachytherapy, with brachytherapy being selected more often in those with improved socioeconomic status. In fact, there is a stronger association between socioeconomic strata and treatment selection than that for race. This particular finding has not to our knowledge been previously reported. However, it does make sense in the context of the previously published literature. In the study from the Massachusetts Cancer Registry, multivariable analysis revealed that those with increasing poverty level, with Medicaid or who were uninsured were less likely to undergo radical prostatectomy and more likely to undergo radiation. It is difficult to ascertain precisely how these factors may affect the choice whether or not to undergo prostate brachytherapy. However, it is certainly possible that in the same fashion that the poverty and/or insurance level seems to impact the decision whether or not radical prostatectomy is chosen, it may also impact in some way the decision to undergo brachytherapy. Furthermore, in a study by Hoffman et al reporting on from the Prostate Cancer Outcomes Study, insurance status did influence the treatment decision whether or not to undergo radiation therapy [13].

We are unable to determine a causal relationship between lower socioeconomic status and differences in the brachytherapy rates in this study. However, the possibility that prostate brachytherapy is being underutilized, particularly in those of lower socioeconomic status, has significant potential implications in the context of the overall cost of care for these patients. Several studies have reported similar outcomes between brachytherapy and external beam radiation, yet at $30 \%$ or lower cost compared to intensity modulated radiation therapy [14-15]. Furthermore, other studies, though with their own limitations, have reported that the incorporation of brachytherapy into the treatment paradigm even improves biochemical outcomes compared to external beam radiation, suggesting the possibility that the lower cost modality may in fact be superior [16-18]. One possible reason for the differences in brachytherapy use is that these racial and socio-economic differences may be correlated with a difference in the availability of brachytherapy as a treatment option in the community setting. The Amer- ican Brachytherapy Society (ABS) has carried out surveys to identify trends in the practice of brachytherapy. The first survey was carried out with data from 1995 [19], and the second used data from 2010 [20]. A comparison of the two surveys shows that the median number of years of experience for low dose rate brachytherapists increased from 5 to 10 years over the course of the 12 years since the preceding survey, indicating that there are fewer new graduates practicing brachytherapy. In addition, compared with the first ABS survey, a smaller proportion of respondents received formal brachytherapy residency training (43\% vs. $56 \%$ ) or formal "hands-on" brachytherapy training (15\% vs. $63 \%$ ). This suggests that newly trained radiation oncologists may not be as experienced with the techniques as those trained in the past. The quality of brachytherapy is highly dependent upon experience, as reflected by a study by Merrick et al. that found that brachytherapists who perform a high volume of cases were much more likely to have excellent target coverage in their implants [21]. Several other studies have also established the presence, and importance of the learning curve in prostate brachytherapy quality [22-25].

It is possible that this reduced access to physicians with brachytherapy experience may be more pronounced in rural or inner city areas with an overall lower economic status. This concept has previously been noted by Steenland et al. [26] In their study they noted that those with higher income were more likely to undergo surgery than radiation regardless of race. They also reported that those in rural areas who did receive treatment were less likely to receive surgery or brachytherapy, and were instead likely to receive EBRT alone. In a study by Denberg et al., 2000 urologists were presented with a clinical vignette of a 70 year old patient with low risk, localized prostate cancer and asked to make a treatment recommendation. Four versions of the vignettes were made, which were identical with the exception of patient race (African American or Caucasian) and socioeconomic status (middle-income and married or low income and widowed). The responses suggested that perceived socioeconomic vulnerability in African American patients more strongly influenced clinicians to avoid radical prostatectomy [27]. The implication is that these factors may have an influence on clinicians, and one wonders if they would similarly impact whether clinicians recommend brachytherapy.

There are several limitations to note in this current SEER analysis. First, the division of patients into socioeconomic quartiles is a relatively arbitrary one. It is based on the reported average cost of living adjusted income of the county in which the patient was treated. However, we do not have individual data regarding their socioeconomic status, thereby making it likely that we unknowingly assigned some individual patients into the wrong socioeconomic group. Second, SEER does not record a variety of potentially confounding comorbidities, such as cardiac, pulmonary and other medical problems, that may have influenced the final decision to undergo brachytherapy as opposed to external beam radiation. Finally, we are reliant on the accuracy of the coding by SEER to correctly identify which treatments were received. However, prior studies have compared the coding for radiation treatments in prostate can- 
cer between SEER and Medicare and have found $93 \%$ concordance indicating that SEER coding is reliable [28].

\section{Conclusions}

Despite some limitations, this SEER analysis does provide the ability to analyze over 38,000 patients across various parts of the United States. We found that those patients with improved socioeconomic status as well as those who are Caucasians are more likely to undergo prostate brachytherapy as a component of their treatment. This may be partially related to reduced access to care due to reduced training and comfort level of radiation oncologists in performing prostate brachytherapy leading to fewer physicians performing this procedure in the community. However, these findings of potential racial and socioeconomic disparities are important both from a potential inequality of care perspective as well as a cost of care perspective. Further studies are needed to both confirm and further elucidate the causes of these disparities, so that potential solutions can be suggested to address these differences.

\section{Acknowledgement}

No research funding was received in association with this research.

\section{References}

1. http://www.cancer.org/cancer/prostatecancer/detailedguide/ prostate-cancer-key-statistics [accessed 7/9/13].

2. Chornokur G, Dalton K, Borysova ME et al. Disparities at presentation, diagnosis, treatment, and survival in African American men, affected by prostate cancer. Prostate 2011; 71: 985-997.

3. Du XL, Fang S, Coker AL et al. Racial disparity and socioeconomic status in association with survival in older men with local/regional stage prostate carcinoma: findings from a large community-based cohort. Cancer 2006; 106: 1276-1285.

4. Cheng I, Witte JS, McClure LA et al. Socioeconomic status and prostate cancer incidence and mortality rates among the diverse population of California. Cancer Causes Control 2009; 20: $1431-1440$.

5. Woods LM, Rachet B, Colema MP. Origins of socio-economic inequalities in cancer survival: a review. Ann Oncol 2006; 17: 5-19.

6. Terakedis BE, Rossi PJ, Liauw SL et al. A surveillance, epidemiology, and end results registry analysis of prostate cancer modality time trends by age. Am J Clin Oncol 2010; 33: 619-623.

7. Zelefsky MJ, Moughan J, Owen J et al. Changing trends in national practice for external beam radiotherapy for clinically localized prostate cancer: 1999 patterns of care survey for prostate cancer. Int J Radiat Oncol Biol Phys 2004; 59: 1053-1061.

8. Guedea F, Venselaar J, Hoskin P et al. Patterns of Care for Brachytherapy in Europe: Updated Results. Radiother Oncol 2010; 97: 514-520.

9. Terakedis BE, Rossi PJ, Liauw SL et al. A surveillance, epidemiology, and end results registry analysis of prostate cancer modality time trends by age. Am J Clin Oncol 2010; 33: 619-623.

10. Hamilton AS, Albertsen PC, Johnson TK et al. Trends in the treatment of localized prostate cancer using supplemented cancer registry data. BJU Int 2011; 107: 576-584.

11. Underwood W, De Monner S, Ubel P et al. Racial/ethnic disparities in the treatment of localized/regional prostate cancer. J Urol 2004; 171: 1504-1507.
12. Rose AJ, Backus BM, Gershman ST et al. Predictors of aggressive therapy for nonmetastatic prostate carcinoma in Massachusetts from 1998 to 2002. Med Care 2007; 45: 440-447.

13. Hoffman RM, Harlan LC, Klabunde CN et al. Racial differences in initial treatment for clinically localized prostate cancer. J Gen Internal Med 2003; 18: 245-253.

14. Hayes J, McMahon P, Pearson SD. Institute For Clinical and Economic Review; Final Appraisal Document. Brachytherapy and proton beamtherapy for treatment of clinically-localized, low risk prostate cancer. Institute for Clinical and Economic Review; available at: http:/ / www.icerreview.org/index.php? option_com_content\&task_view\&id_18\&Itemid_39 (accessed 06/11/2013).

15. Shah C, Lanni TB, Ghilezan MI et al. Brachytherapy provides comparable outcomes and improved cost-effectiveness in the treatment of low/intermediate prostate cancer. Brachytherapy 2012; 11: 441-445.

16. Hoskin PJ, Motohashi K, Bownes P, et al. High dose rate brachytherapy in combination with external beam radiotherapy in the radical treatment of prostate cancer: initial results of a randomized phase three trial. Radiother Oncol 2007; 84: 114-120.

17. Pieters BR, de Back DZ, Koning CC et al. Comparison of three radiotherapy modalities on biochemical control and overall survival for the treatment of prostate cancer: a systematic review. Radiother Oncol 2009; 93: 168-173.

18. Grimm P, Billiet I, Bostwick D et al. Comparative analysis of prostate-specific antigen free survival outcomes for patients with low, intermediate and high risk prostate cancer treatment by radical therapy. Results from the Prostate Cancer Results Study Group. BJU Int 2012; 109: 22-29.

19. Prestidge BR, Prete JJ, Buchholz TA et al. A survey of current clinical practice of permanent prostate brachytherapy in the United States. Int J Radiat Oncol Biol Phys 1998; 40: 461-465.

20. Buyyounouski MK, Davis BJ, Prestidge BR et al. A survey of current clinical practice in permanent and temporary prostate brachytherapy: 2010 Update. Brachytherapy 2012; 11: 299-305.

21. Merrick GS, Lief JH, Grimm P et al. The effect of pro-quora case volume on post-implant dosimetry. Int J Radiat Oncol Biol Phys 2011; 81: 727-734.

22. Lee WR, Deguzman AF, Bare RL et al. Postimplant analysis of transperineal interstitial permanent prostate brachytherapy: Evidence for a learning curve in the first year at a single institution. Int J Radiat Oncol Biol Phys 2000; 46: 83-88.

23. Bladou F, Salem N, Simonian-Sauve M et al. Permanent iodine 125 implant brachytherapy in localized prostate cancer: Results of the first 4 years of experience. Prog Urol 2004; 14: 345-352.

24. Hoinkis C, Hakenberg OW, Lehmann D et al. Evaluation of dose-volume histograms after prostate seed implantation: 4-year experience. Strahlenther Onkol 2004; 180: 550-556.

25. Bice WS Jr., Prestidge BR, Grimm PD et al. Centralized multiinstitutional postimplant analysis for interstitial prostate brachytherapy. Int J Radiat Oncol Biol Phys 1998; 41: 921-927.

26. Steenland K, Goodman M, Liff J et al. The effect of race and rural residence on prostate cancer treatment choice in rural Georgia. Urology 2011; 77: 581-587.

27. Denberg TD, Kim FJ, Flanigan RC et al. The influence of patient race and social vulnerability on urologist treatment recommendations in localized prostate carcinoma. Med Care 2006; 44: 1137-41.

28. Virnig BA, Warren JL, Cooper GS et al. Studying radiation therapy using SEER-Medicare-linked data. Med Care 2002; 40 (8 Suppl IV): 49-54. 